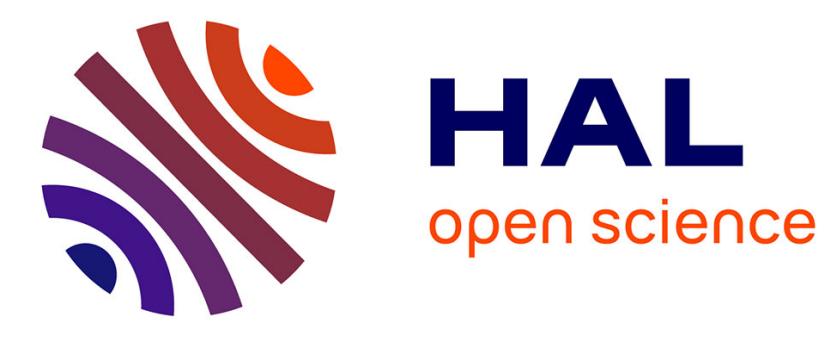

\title{
Oppositions in Fuzzy Linguistic Summaries
}

Gilles Moyse, Marie-Jeanne Lesot, Bernadette Bouchon-Meunier

\section{To cite this version:}

Gilles Moyse, Marie-Jeanne Lesot, Bernadette Bouchon-Meunier. Oppositions in Fuzzy Linguistic Summaries. FUZZ-IEEE'15 - IEEE International Conference on Fuzzy Systems, Aug 2015, Istanbul, Turkey. pp.1-8, 10.1109/FUZZ-IEEE.2015.7337863 . hal-01168061

\section{HAL Id: hal-01168061 https://hal.science/hal-01168061}

Submitted on 19 Aug 2015

HAL is a multi-disciplinary open access archive for the deposit and dissemination of scientific research documents, whether they are published or not. The documents may come from teaching and research institutions in France or abroad, or from public or private research centers.
L'archive ouverte pluridisciplinaire HAL, est destinée au dépôt et à la diffusion de documents scientifiques de niveau recherche, publiés ou non, émanant des établissements d'enseignement et de recherche français ou étrangers, des laboratoires publics ou privés. 


\title{
Oppositions in Fuzzy Linguistic Summaries
}

\author{
Gilles Moyse, Marie-Jeanne Lesot, Bernadette Bouchon-Meunier \\ Sorbonne Universités, UPMC Univ Paris 06, UMR 7606, LIP6, F-75005, Paris, France \\ CNRS, UMR 7606, LIP6, F-75005, Paris, France \\ gilles.moyse@lip6.fr
}

\begin{abstract}
An important aspect of interpretability in Fuzzy Linguistic Summaries (FLS) is the absence of opposition therein, which is not guaranteed by the the current approaches used for their generation, possibly leading to confusion for the end-user. In this paper, we first introduce a 3-level hierarchy to organise the models of opposition starting from simpler sentences, then enriched with generalised quantifiers and thirdly considering the several negation operators allowed by fuzzy logic. We then introduce a general model of opposition for FLS sentences, which we propose to represent as a 4-dimensional cube. We additionally discuss the antonym property in this analysis framework and prove it for general protoforms.
\end{abstract}

Index Terms-Fuzzy Linguistic Summaries, Interpretability, Consistency, Square of Opposition, Antonymy

\section{INTRODUCTION}

Fuzzy Linguistic Summaries (FLS) [1], [2] can be defined as texts made of several sentences describing distinct characteristics of a given dataset. Their interpretability [3], [4] is a central issue, related more specifically to the one of consistency, i.e. absence of contradiction. Indeed, standard FLS [1], [5], [6] do not ensure that opposed sentences, e.g. "Most young people are short" and "Most young people are tall", cannot be returned within the same summary.

In order to prevent them, such oppositions must first be identified, which is not trivial due to the numerous degrees of freedom allowed in the generation of these sentences, as discussed in this paper. It furthermore proposes a formalism detailing these oppositions and a representation as a 4-dimensional cube.

The paper is organised as follows: first, a reminder about FLS and in particular their truth evaluation is given in Section II. Sections III to V describe the proposed hierarchical view of the opposition issue, presenting existing models describing oppositions between sentences, organised according to their generality, i.e. having increasing degrees of freedom: Section III describes formalisms allowing to represent opposition between sentences, either simple or quantified using only $\forall$ and $\exists$. Section IV considers the case of sentences built using generalised quantifiers. Section V details a third degree of freedom present in sentences composing FLS, namely the use of different kinds of negation in fuzzy logic. In Section VI, we propose to study opposition in FLS at a general level, by first defining the parts of sentences over which negations can be applied, then detailing the different types of sentences which can be generated using all the degrees of freedom introduced in Sections III to $\mathrm{V}$. It proposes the 4-dimensional cube of oppositions that constitutes a graphical representation of all relations holding between all variants of negations that can be built using the various degrees of freedom existing in FLS. Finally, the relation of duality is discussed in Section VII, allowing to prove a specific property of FLS so far satisfied for simple protoforms only.

\section{FUZZY LinguistiC SUMMARIES}

\section{A. Protoform Definition}

A Fuzzy Linguistic Summary (FLS) is a set of sentences describing data [1], [2], [5]-[7]. Each sentence is a couple made of an instantiated protoform, which is a sentence schema, and a truth degree $t$, measuring the protoform adequacy with the data. In the case of people described by their age and height, such a sentence can be illustrated by the example "Less than 5 young people are short" $(t=0.83)$.

The protoforms considered in this paper are " $Q R x$ are $P$ ", where $x$ denotes the studied data, $Q$, $R, P$ are respectively called quantifier, qualifier and summariser. In the previous example, $Q$ is LessThan 5, $R$ is Young, $P$ is Short and $x$ is a list of people.

In a general setting, $Q$ is modelled by a fuzzy set, denoting either an absolute quantifier as About10 or MoreThan5, or a relative one as LessThanHalf or Most [6]. $R$ and $P$ are also fuzzy sets, representing modalities of linguistic variables [8].

\section{B. Truth Value Evaluation}

Though alternative proposals exist for protoform truth value evaluation (see e.g. [7], [9]-[13]), this paper focuses on the standard one, which consists in a three step process to compute the truth value of the protoform " $Q R x$ are $P$ " [1].

First, the membership of each data $x$ to $R$ and $P$ is computed through their membership functions, denoted $R(x)$ and $P(x)$ respectively.

Then, the number of $x$ being both $R$ and $P$ is evaluated through the counting function $\nu$ :

$$
\nu(R, P)=\sum_{x \in X} \top(R(x), P(x))
$$

where $T$ is a t-norm. $\nu$ is the fuzzy cardinality of the fuzzy intersection of $R$ and $P$. Indeed, the fuzzy cardinality is defined for a fuzzy set $A$ as $|A|=\sum_{x} A(x)$.

The third step is the quantification of the count returned by $\nu$, i.e. the membership of this count to $Q$. Absolute 
quantifiers are usually defined over $\mathbb{R}^{+}$, to quantify any positive value, while relative quantifiers are defined on $[0,1]$ to express ratios. Hence, in the case of absolute quantifiers, the count returned by $\nu$ is used as is, whereas in the case of relative ones, it is divided by the size of the considered universe $R$, computed as its fuzzy cardinality.

So as to take into account the two types of quantifiers, a normalising function $\rho$ is introduced:

$$
\rho(R, Q)= \begin{cases}1 & \text { if } Q \text { is absolute } \\ 1 /|R| & \text { if } Q \text { is relative }\end{cases}
$$

The general expression of the truth value is then:

$$
t(Q R x \text { are } P)=Q(\rho(R, Q) \cdot \nu(R, P))
$$

\section{Proposed Hierarchical View of the Opposition Issue}

The computation of the truth value defined in Eq. (3) takes into account the whole dataset, but not the other sentences. It thus does not guarantee that truth values numerically reflect opposition between sentences, where actually both the notions of opposed sentences and their expected truth values are to be defined and justified. As discussed in this paper, the various parameters implied in the truth evaluation show how complex the detection of opposite sentences is.

Even though a clear definition of opposite sentences in the context of FLS has not been exposed yet, the question has been raised several times and solved in simpler paradigms, detailed in the three next sections: they present how opposition is identified in increasingly complex frameworks, i.e. allowing sentences to be built with a growing number of degrees of freedom. In Section III, oppositions are represented for simple and quantified sentences. The only degree of freedom for these sentences relies on the use or not of the quantifiers All or Some.

However, FLS allow richer and more complex generalised quantifiers to be employed as a second degree of freedom, detailed in Section IV. The different adaptations of the representations of opposition designed to take into account these quantifiers are also presented.

Then, Section V details a third degree of freedom linked to the use of different negations in fuzzy logic, namely antonym and complement. The representation of oppositions in this broader context has not been given yet and is proposed in Section VI.

\section{III. $1^{\text {st }}$ LeVel: Simple AND QUANTIFIED Sentences}

The simplest kind of opposition, studied in the first subsection, is the one between two simple sentences usually denoted " $S$ is $P$ " and " $S$ is $P$ ", with $S$ a subject and $P$ and $P^{\prime}$ two predicates, e.g. "John is tall" and "John is short". In this formalism, $S$ denotes an individual and such a sentence corresponds to a simplified protoform " $x$ is $P$ ", with neither quantifier nor qualifier. It is then extended to two specific quantifiers to study the opposition between quantified sentences of the form "All $S$ are $P$ " or "Some $S$ are P", yielding its representation as squares, as detailed in the second and third subsections.

Lastly, more elaborate proposals regarding the opposition between both simple and quantified sentences are presented in the last subsection.

\section{A. Opposition of Simple Sentences}

The earliest study of opposition for simple sentences dates back to Aristotle and relies on the two laws of Excluded Middle (EM) and Non Contradiction (NC) [14, p. 62]. EM states that " $S$ is $P$ or not $P$ " and NC that "No $S$ is $P$ and not $P$ ".

From these two laws, Aristotle introduce three classical relations of opposition for simple sentences of the form " $S$ is $P$ ", namely contradiction, contrary and subcontrary: given two predicates $P$ and $P^{\prime}$, " $S$ is $P$ " and " $S$ is $P^{\prime \prime}$ " are in contradiction if $S$ must be either $P$ or $P^{\prime}$ but not both. For instance, an object is either $P=$ cold or $P^{\prime}=$ not cold, not both. Therefore, two predicates in a contradiction relation both satisfy EM, since $S$ has to be $P$ or $P^{\prime}$, and NC, since $S$ cannot be $P$ and $P^{\prime}$.

The contrary relation is satisfied if $S$ cannot be both $P$ and $P^{\prime}$ but may be neither: e.g. an object cannot be both $P=$ hot and $P^{\prime}=$ cold, but can be lukewarm. So two predicates in a contrary relation satisfy NC but not EM.

The subcontrary relation is satisfied if $S$ must be either $P$ or $P^{\prime}$, possibly both: e.g. an object has to be either $P=$ not hot or $P^{\prime}=$ not cold, and can be both if it is lukewarm. Hence, two predicates in a subcontrary relation satisfy EM but not NC.

\section{B. Classical Square of Opposition}

Based on the three relations defined for simple sentences, the classical or Aristotelian square of oppositions has been proposed for quantified sentences, considering the quantifiers All and Some, yielding the four sentences "All $S$ are P", "No $S$ is P", "Some $S$ are $P$ " and "Some $S$ are not $P$ ". It must be underlined that a shift in the meaning of $S$ occurs in this classic notation: $S$ here becomes a predicate that an individual $x$ can satisfy or not. These sentences can be interpreted using the protoform notations detailed in Section II-A with the quantifiers All, None or Some, the qualifier $S$ and the summariser $P$ or not $P$.

Their oppositions are modelled in the square illustrated on Fig. 1. Corners $A / E$ satisfy the contrary relation since "All $S$ are $P$ " and "No $S$ is $P$ " cannot be true but may be false together. Similarly, $A / O$ and $E / I$ are in contradiction, and $I / O$ satisfy the subcontrary relation.

An additional relation, called subaltern, is also present in the square. It is an implication, not an opposition: "All $S$ are $P$ " implies that "Some $S$ are $P$ ", and "No $S$ are $P$ " that "Some $S$ are not $P$ ".

For instance, if $S$ is a person and $P$ his or her tallness, then the corner $A$ "All persons are tall" satisfies a contradiction relation with $O$ "Some persons are not tall", a contrary one 


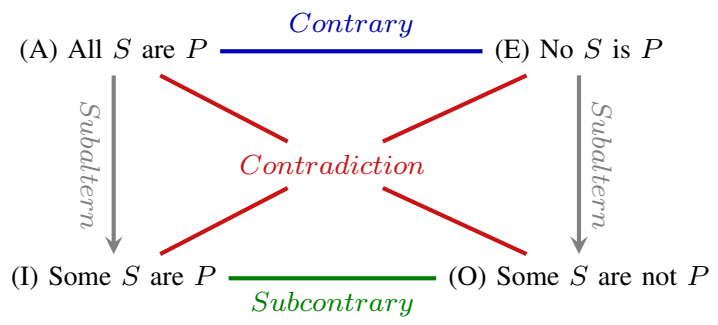

Figure 1. Classical or Aristotelian square of opposition

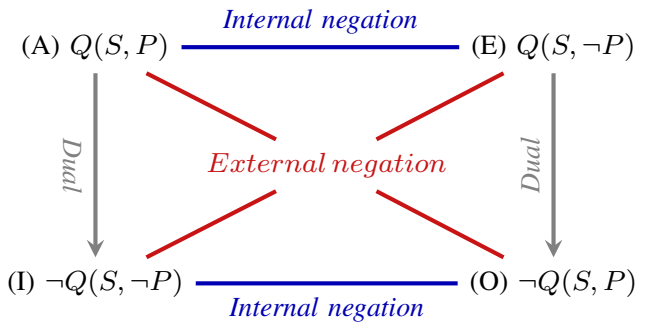

Figure 2. Modern square of opposition

with $E$ "No person is tall" and a subaltern or implication one with I "Some persons are tall".

\section{Modern Square of Opposition}

The modern square (see Fig. 2) also models opposition between quantified sentences, but differs from the classical one in three respects [15]. First, it is generally formalised using the notation $Q(S, P)$ to represent " $Q S$ are $P$ ". It has as a specific case "All $S$ are $P$ " for $Q=A l l$. Second, it uses of a general quantifier $Q$ which can differ from All or Some. Third, it considers 3 rather than 4 relations: the internal negation which is the negation of the predicate $P$, and the external negation, which is the negation of the whole sentence. The dual relation is defined as the commutative composition of an internal and an external negation.

Thus, the modern square is more expressive than the classical one since it allows quantifiers different from $\operatorname{All}(\forall)$ and Some ( $\exists$ ) to be used. Moreover, if $Q$ is $\forall$ and $\neg Q$ is $\exists$, it is equivalent to the classical square, as illustrated on Fig. 3 with a modern formalism: e.g. the $A$ corner standing for "All $S$ are $P$ " is formally written $\forall x, S(x) \rightarrow P(x)$ (the parentheses are omitted in the following to lighten the formalism), using a logical implication between the predicates $S$ and $P$ and making the involved individuals $x$ explicit. The internal negation of $A$, yielding the $E$ corner, is computed by applying a negation on $P: \forall x, S x \rightarrow \neg P x$, read "No $S$ is $P$ ". The external negation of $A$ is $O$, obtained with a negation over the entire expression, yielding $\neg(\forall x, S x \rightarrow P x)=\exists x, \neg(\neg S x \vee P x)=\exists x, S x \wedge \neg P x$, read "Some $S$ are not $P$ ". The last corner $I$ is deduced with an internal negation over $O$, yielding $\exists x, S x \wedge P x$, read "Some $S$ are P".

Clearly, the classical relations are verified in this square: the contrary relation holds for the corners $A / E$, contradiction

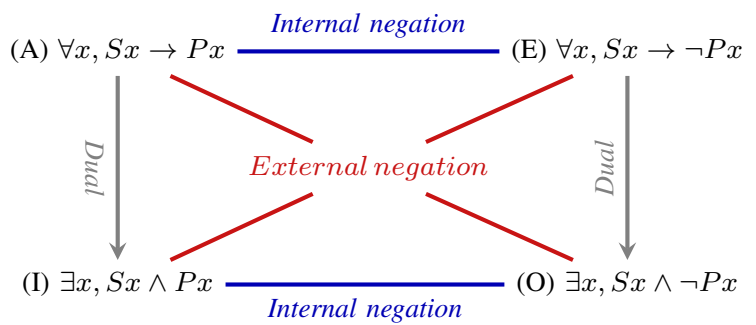

Figure 3. Modern square for $Q=\forall$

(U)

$\forall x,(S x \rightarrow P x) \vee \forall y,(S y \rightarrow \neg P y)$

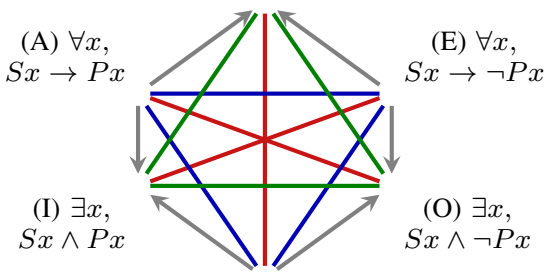

(Y)

$$
\exists x,(S x \wedge P x) \wedge \exists y,(S y \wedge \neg P y)
$$

Figure 4. Hexagon of opposition [16]

for $A / O$ and $E / I$, subcontrary for $I / O$, and subaltern for $A / I$ and $E / O$. As shown in Section IV, this is not the case for any quantifier $Q$.

\section{Other Structures of Opposition}

a) Interpretation of "Some": Beside the extension of the square to other quantifiers described above, other interpretations of $\forall$ and $\exists$ have been proposed, yielding more complex shapes than the square. In particular, in the previously mentioned squares, Some is interpreted as "Some, maybe all". However, it may be understood as "Some, but not all". The hexagon of opposition [16] illustrated on Fig. 4 is defined using this stricter definition. It adds two corners to the classical square: $Y$, interpreted as "Neither all nor none", and $U$, "All or none". The three classical relations are satisfied with these two new corners.

As the three mutually exclusive cases $A, E$ and $Y$ are such that $A \wedge E$ and $A \wedge Y$ and $E \wedge Y$ are false and $A \vee E \vee Y$ is a tautology, they can be represented as an hexagon [17].

b) Simple Sentences with More Than Two Terms: Another direction of extension aims at considering terms different from $P$ or not $P$ for simple sentences. A geometrical method to design shapes satisfying the classical relations is proposed in [18] for any set of terms building a partition of the universe in a set of predicates $P_{1}, \ldots, P_{n}$ such that for all $x, P_{1}(x) \vee \ldots \vee P_{n}(x)$ is true and $\forall i, j, P_{i}(x) \wedge P_{j}(x)$ is false. In this method, each element $P_{i}$ of the partition is linked to the others through a contrary relation; its contradictory term $\neg P_{i}$ is located symmetrically with respect to the centre of the shape; subcontrary terms are identified as the contradiction of contrary terms and linked with one another, 


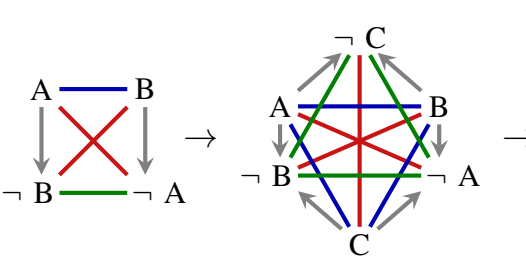

Square: 2 terms
Hexagon: 3 terms

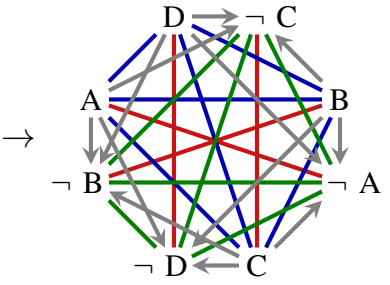

2D-octagon or 3D-cube:

4 terms

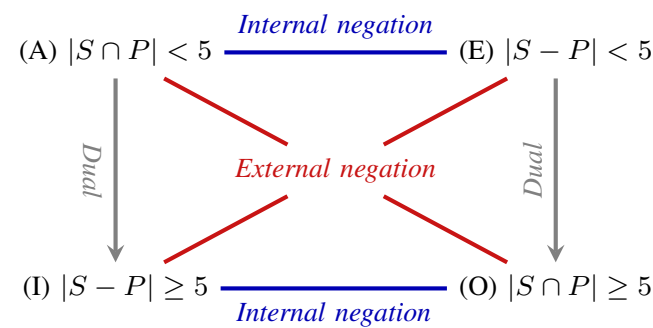

Figure 6. Modern square of LessThan5 $(S, P)$
Figure 5. $n$-partition polygons maintaining the 4 relations of the classical square [18]

and adjacent corners are linked with a subaltern relation [18]. Fig. 5 illustrates the construction of shapes containing an increasing number of terms and maintaining the classical relations. In the cube, represented in the $2 \mathrm{D}$-figure as an octagon, $\{A, B, C, D\}$ is a partition of the universe. Hence, considering the $A$ vertex, contrary relations hold with $B$, $C, D$, contradiction with $\neg A$ and subalterns with $\neg B, \neg C$ and $\neg D$. The subcontrary relation holds for vertices with a negation, as e.g. between $\neg A$ and $\neg B, \neg C$ and $\neg D$.

E.g. considering the colour of an object, $A$ for Red and $B$ for Green in the square, if the object is Red then it is not Green, as the subaltern relation from $A$ to $B$ illustrates, and it cannot be not Red as shown by the contradiction relation between $A$ and $\neg A$. With $C$ for Blue and the hexagon, if an object is Red then it is not Green and not Blue as indicated by the subaltern relations from $A$ to $B$ and from $A$ to $C$ and so on for the other relations and figures.

\section{IV. $2^{\text {nd }}$ LEVEL: Generalised QuANTIFIERS}

In this section, a new degree of freedom is added through generalised quantifiers, presented in the next subsections, with their semantics and their links with the logical squares.

\section{A. Generalised Quantifiers}

Generalised quantifiers [19] allow to express intermediary states between $\forall$ and $\exists$. In a linguistic context, the generalised quantifier LessThan5 $(S, P)$ for instance is read "Less than $5 S$ are $P$ ", and its truth value is evaluated as the truth value of an expression using standard set operators, as LessThan5 $(S, P) \Leftrightarrow|S \cap P|<5$. Other examples of set interpretation of quantifiers include [20]:

$$
\begin{aligned}
\operatorname{All}(S, P) & \Leftrightarrow S \subseteq P \\
\operatorname{No}(S, P) & \Leftrightarrow S \cap P=\varnothing \\
\operatorname{MoreThan20\% }(S, P) & \Leftrightarrow|S \cap P| /|S|>0.2 \\
\operatorname{Most}(S, P) & \Leftrightarrow|S \cap P|>|S-P|
\end{aligned}
$$

The set notations used in this context can be compared to the propositional ones used for the modern square, e.g. for $\operatorname{All}(S, P), S \subseteq P$ applying to the predicate interpretation, is equivalent to $\forall x(S x \rightarrow P x)$.

\section{B. Links with Logical Squares}

Generalised quantifiers can be used directly within the modern square of opposition [15] since it is designed with a generic quantifier $Q$, as opposed to the classical one using "All", "Some", and "No". For instance the corners of the square illustrated on Fig. 6 are built using the definitions of internal and external negations for the quantifier LessThan5.

It must be remarked that these definitions do not satisfy the relations of the classical square [21]: on Fig. 6, the subcontrary relation does not hold between the corners $I$ and $O$ since they can be both false (if $4 S$ are $P$ and $4 S$ are not $P$ for instance).

Several proposals have been made in order to include generalised quantifiers in the classical square. Two squares, one with Few, Many and the other with Many, Most are proposed [22] but they require several prerequisites to satisfy the classical relations: Many must be interpreted as the contradiction of Most in one case and of Few in the other; furthermore, Many must account for less than $50 \%$ of the elements for the subcontrary to hold.

In the framework of fuzzy type theory, intermediate quantifiers [23] are used to model several generalised quantifiers satisfying the classical relations. As detailed in [24], they are classical $\forall$ or $\exists$ quantifiers whose universe of quantification can be modified in an imprecise way.

Hence, generalised quantifiers, enhancing the expressive power of quantified sentences and adding a degree of freedom in their construction, also make oppositions more difficult to formalise since they do not systematically satisfy the classical relations.

\section{V. $3^{r d}$ LEVEL: FuZZY NEGATIONS}

Simple and quantified sentences have been presented in the previous sections using classical logic, equipped with the single negation operator $\neg$. Fuzzy logic though allows more than one negation, yielding an additional degree of freedom in the constitution of sentences in FLS. In this notation, the predicates involved in the considered sentences are thus considered as fuzzy predicates associated with a truth degree in $[0,1]$ and represented by their membership functions.

In the following, $A$ denotes a fuzzy set with a membership function defined from $\left[a^{-}, a^{+}\right]$to $[0,1] . A(x)$ represents the degree to which " $x$ is $A$ ", to be compared to the sentence " $S$ is $P$ " from Section III-A with $S=x$ and $P=A$. 
After reminding the classical fuzzy negation operator, this section discusses the three negations defined for predicates in fuzzy logic, namely complement, antonym and antonym complement as well as their links with the classical relations.

\section{A. Negation Operator}

Given an interval $I=\left[i^{-}, i^{+}\right]$, the fuzzy negation operator $n$ is defined as:

$$
\forall y \in I, n(I, y)=i^{+}-i^{-}-y
$$

Hence, the negation is an involution from $I$ to $I$, corresponding to an axial symmetry with respect to the axis $y=\left(i^{+}+i^{-}\right) / 2$. In the following, when $I$ is clearly defined, $n(I, y)$ is written $n(y)$ or equivalently $\bar{y}$.

\section{B. Complement}

For any fuzzy set $A$, its complement $\bar{A}$ is defined as:

$$
\bar{A}(x)=n([0,1], A(x))=1-A(x)
$$

i.e. the negation of the membership function $A(x)$, defined in $[0,1]$.

The fuzzy complement is the direct counterpart of the classical negation, equal to the latter for a crisp set. It is also involutive, i.e. $\overline{\bar{A}}=A$.

As illustrated on Fig. 7, $\bar{A}$ is symmetric to $A$ with respect to the horizontal axis $y=1 / 2$.

It must be noted that, although usually $A \neq \bar{A}$, there exists a fuzzy set such that $A=\bar{A}$, which may make this opposition relation counterintuitive. Yet, this fuzzy set is defined by the membership function $A(x)=1 / 2$ for all $x \in\left[a^{-}, a^{+}\right]$, which is an uninteresting fuzzy set in a linguistic context and is not likely to occur in FLS.

\section{Antonym}

For any fuzzy set $A$, its antonym $\widehat{A}$ is defined as:

$$
\widehat{A}(x)=A\left(n\left(\left[a^{-}, a^{+}\right], x\right)\right)=A\left(a^{+}-a^{-}-x\right)=A(\bar{x})
$$

i.e. the negation of the parameter $x$ defined in $\left[a^{-}, a^{+}\right]$. It is involutive, i.e. $\hat{\hat{A}}=A$.

As illustrated on Fig. $8, \widehat{A}$ is symmetric to $A$ with respect to the vertical axis $x=\left(a^{+}-a^{-}\right) / 2$.

It can be underlined, again, that although in most cases $A \neq \widehat{A}$, there exist fuzzy sets such that $A=\widehat{A}$ : in a Ruspini partition made of an odd number of equally sized modalities, the central modality is equal to its antonym, as illustrated by $B$ on Fig. 8 .

\section{Antonym Complement}

The antonym complement (a.c.) $\widehat{\bar{A}}$ is the composition of an antonym and a complement and is defined as:

$$
\widehat{\bar{A}}(x)=1-A\left(a^{+}-a^{-}-x\right)=\bar{A}(\bar{x})
$$

It is commutative, so $\widehat{\bar{A}}=\overline{\widehat{A}}$ [25]. Furthermore, if $A=\widehat{\bar{B}}$, then $\widehat{A}=\bar{B}, \bar{A}=\widehat{B}, \widehat{\bar{A}}=B$.

As illustrated on Fig. 9 and 10, $\widehat{\bar{A}}$ is symmetric to $A$ with respect to the point $\left(\left(a^{+}-a^{-}\right) / 2,1 / 2\right)$.

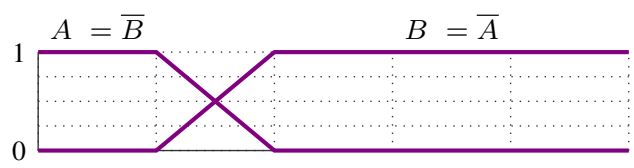

Figure 7. $A$ and $B$ are complements

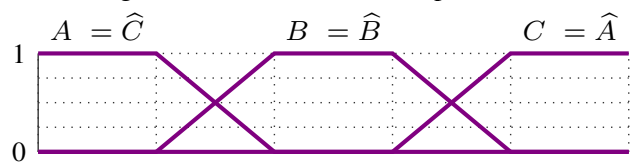

Figure 8. $\quad A$ and $C$ are antonyms, $B$ is self antonym

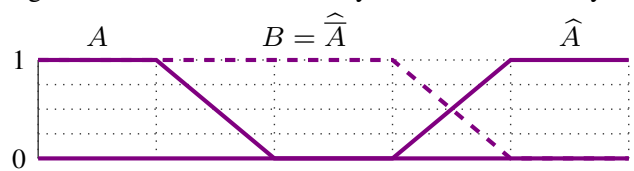

Figure 9. $\quad A$ and $B$ (dashed) are antonym complements

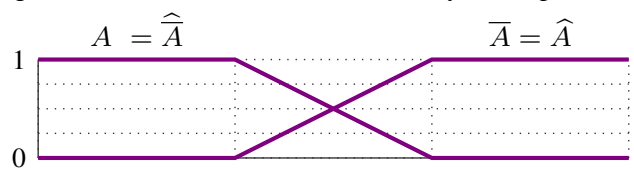

Figure 10. $A$ is a self antonym complement

\section{E. Establishing Links Between Classical Relations and Fuzzy Negations}

In order to study the relations between the three fuzzy negations discussed above (complement, antonym and antonym complement), and the classical relations (contradiction, contrary, subcontrary and subaltern), the Non Contradiction and the Excluded Middle must be defined in a fuzzy context. We propose two variants for them: a strict one, associating $\top(A, \neg A)=0$ with $\mathrm{NC}$ and $\perp(A, \neg A)=1$ with EM, and a soft one, defining $\top(A, \neg A) \leq 0.5$ for $\mathrm{NC}$ and $\perp(A, \neg A) \geq 0.5$ for EM. We propose in this paper to use the soft variant since it is semantically consistent with the two laws and allows to satisfy interesting properties under the general assumptions that the used t-norm and t-conorm are dual and that the considered modalities of linguistic variables define Ruspini partitions.

In this context, different properties are indeed easily provable. First, the fuzzy complement behaves as a classical contradiction since it satisfies both NC and EM. Indeed, due to the properties of Ruspini partitions, $\min (A, \bar{A}) \leq 0.5$ and thus $\top(A, \bar{A}) \leq 0.5$, hence satisfying NC. Moreover, as $\top(A, \bar{A})=1-\perp(\bar{A}, A)$, so $\perp(A, \bar{A}) \geq 0.5$, thus satisfying EM. Linguistically, the labels for complement modalities can be Hot / not Hot or Few / not Few.

Second, the fuzzy antonym behaves as a classical contrary since it satisfies NC under the constraint that both $A$ and $\widehat{A}$ are modalities of an underlying linguistic variable. Indeed, the Ruspini partition properties directly entail that $\top(A, \widehat{A})=0$ if $A$ and $\widehat{A}$ are not adjacent, and $\top(A, \widehat{A}) \leq$ 0.5 if they are, thus satisfying $\mathrm{NC}$ in both cases. It is however trivially not satisfied if $A=\widehat{A}$, which is the case with the central modality of a uniform Ruspini partition for instance. 
Linguistically, the labels for antonym modalities can be Hot I Cold or Most / Few.

Third, the fuzzy antonym complement behaves as a classical subcontrary with $\bar{A}$ : indeed, since $T(A, \widehat{A}) \leq 0.5$, $\perp(\bar{A}, \widehat{\bar{A}}) \geq 0.5$ so $\bar{A}$ and $\widehat{\bar{A}}$ satisfy EM. Linguistically, the labels for antonym complement modalities can be Hot / not Cold or Most / not Few.

Lastly, the fuzzy antonym complement satisfies an implication relation, in a similar way as in the modern square. Indeed, in a Ruspini partition, $\widehat{A}(x) \leq \bar{A}(x)$, so $A(x) \leq \widehat{\bar{A}}(x)$. So, denoting $I_{g}(x, y)$ and $I_{\Delta}(x, y)$ the Gödel and Goguen fuzzy implications respectively, $I_{g}(A, \widehat{\bar{A}})=I_{\Delta}(A, \widehat{\bar{A}})=1$. For instance, the pair Hot / not Cold, can be seen as an implication, i.e. if it is hot, then it is not cold, or with a pair of quantifiers, if "Most $S$ are $P$ " then "not Few $S$ are $P$ ". If the antonym and the complement, usually different, are equal, then $A=\widehat{\bar{A}}$, and $A$ is a.c. with itself, implying that $A$ and $\widehat{\bar{A}}$ partition the whole universe of discourse (see Fig. 10).

Thus, negations in fuzzy logic allow to satisfy the classical relations for simple sentences. The next section discusses the matter of quantified ones and thus proposes a study in the general case of FLS, integrating all degrees of freedom.

\section{Proposed General Model of Opposition}

In this section, opposition between quantified sentences used in FLS is studied. First, the different negation protoforms are presented, followed by the operations which can be applied on them, based on the fuzzy negations. Their representation as quadruplets is next introduced, and finally, the 4-cube of opposition showing the relations between the negation protoforms is introduced.

\section{A. Negation Protoforms}

As reminded in Section II, a protoform " $Q R x$ are $P$ " is made of a quantifier $Q$, a qualifier $R$ and a summariser $P$. A negation holds between two protoforms only if they relate to the same universe of discourse, i.e. if they share the same qualifier $R$. For instance, "Most young are short" is a negation of "Most young are tall", since they refer to the same universe of discourse, the young persons. Conversely, it is not a negation of "Most elder are short", since the sentences relate to different universes of discourse.

Therefore, the two parts over which a negation can be applied to define a negation for a protoform are the quantifier $Q$ and the summariser $P$. For this reason, protoforms are denoted $Q P$ only in the sequel.

The different combinations of fuzzy set negations applied over $Q P$ allow to define the 16 possible negation protoforms: $Q P, Q \widehat{P}, Q \bar{P}, Q \widehat{\bar{P}}, \widehat{Q} P, \widehat{Q} \widehat{P}, \widehat{Q} \bar{P}, \widehat{Q} \bar{P}, \bar{Q} P, \bar{Q} \widehat{P}$, $\bar{Q} \bar{P}, \bar{Q} \widehat{\bar{P}}, \widehat{\bar{Q}} P, \widehat{\bar{Q}} \widehat{P}, \widehat{\bar{Q}} \bar{P}, \widehat{\bar{Q}} \frac{\hat{P}}{\text {. }}$

It can be noted that some of these negation protoforms may be equal depending on the definitions of $Q$ and $P$. For instance, if $Q=\widehat{Q}$, then obviously $Q P=\widehat{Q} P$.

\section{B. Negation Protoforms Representation}

The 16 forms of protoforms listed above can be obtained from one another combining operations applying the complement of the quantifier or the summariser, respectively denoted $c_{1}$ and $c_{2}$, or the antonym of the quantifier or the summariser, respectively denoted $a_{1}$ and $a_{2}$ : for instance, $a_{1}\left(a_{2}\left(c_{2}(\bar{Q} \bar{P})\right)\right)=\widehat{\bar{Q}} \widehat{P}$. Due to commutativity, operations can be applied in any order.

It can be noted that due to the involutivity and commutativity of these operations, all the 16 negations protoforms can be generated using at most 4 operations starting from any of them.

The specific combinations $a_{1} \circ c_{1}$ and $a_{2} \circ c_{2}$ represent the antonym complement operations on the quantifier and the summariser and are denoted $a c_{1}$ and $a c_{2}$ respectively. The combination $a_{1} \circ c_{2}$, called duality, is discussed in Section VII.

The 16 forms of negation protoforms can also be represented as quadruplets $(a, b, c, d)$ in $\{0,1\}^{4}$, where each component equals 1 if a given operation is applied, starting from $Q P$. $a$ stands for applying $a_{1}, b$ for $c_{1}, c$ for $a_{2}$ and $d$ for $c_{2}$. For instance, $(1,0,0,1)$ corresponds to $a_{1}\left(c_{2}(Q P)\right)$, so $\widehat{Q} \bar{P}$. The notation is allowed since antonym and complement are commutative.

This notation also allows to write the truth value defined by Eq. (3) as, for any protoform $p=(a, b, c, d)$ :

$$
\begin{aligned}
t(p)=\mid b-Q(\mid a-\rho & (R, Q) \times \\
& \nu(R(x),|d-P(|c-x|)|) \mid) \mid
\end{aligned}
$$

$x$ is explicitly denoted in the parentheses of the $\nu$ function so as to take into account $c$.

\section{The Proposed 4-cube of Oppositions}

The figure of oppositions between the quantified sentences in FLS must thus include 16 vertices whose quadruplet representation indicates they are the corners of a 4-dimensional cube, thus yielding a more complex graphic as the figures discussed in Sections III and IV. Now, a classic representation in 3 dimensions of a 4-dimensional cube is a pair of nested cubes, as illustrated on Fig. 11.

Coloured edges link the 16 vertices. Thick edges represent the four basic negation operations, in green for antonyms, red for complements: vertical edges correspond to $c_{1}$, horizontal ones to $a_{2}$, depth ones to $a_{1}$. Thin edges going from one cube to another represent $c_{2}$, thus rendering the fourth dimension. Blue and orange thin edges represent composed operations, respectively antonym complement and duality $d$, discussed in the next section.

56 edges are present in the 4-cube of oppositions: 7 operations $\left(a_{1}, a_{2}, c_{1}, c_{2}, a c_{1}, a c_{2}, d\right)$ over 16 vertices yield $7 \times 16=112$ directed links. Since each operation is involutive, each edge is symmetric, dividing by two the number of links. 


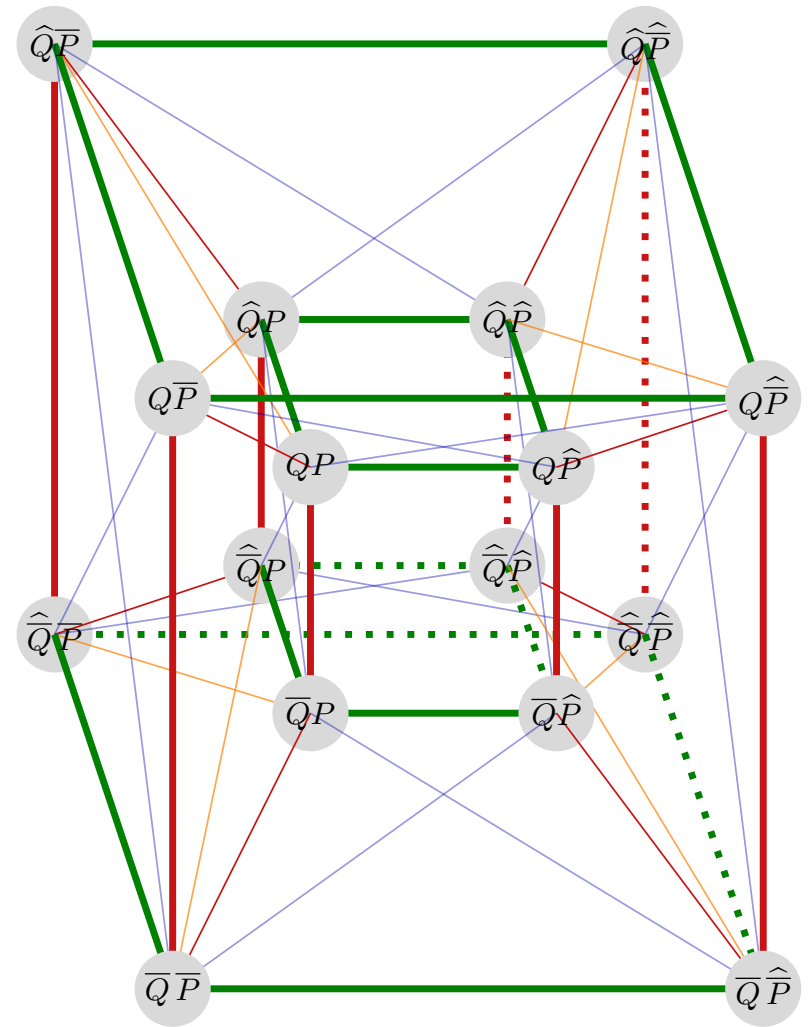

Figure 11. The proposed 4-cube of oppositions

\section{Relations with the Modern Square of Opposition}

As established in Subsection V-E, non quantified sentences with fuzzy negations have several links with the classical square of opposition represented on Fig. 1. In this subsection, we show that quantified protoforms used in FLS and their 4-cube representation have links with the modern square of opposition represented on Fig. 2.

In the modern square, only two operations can be considered, namely internal and external negation: the corresponding protoforms can be represented as couples $(\alpha, \beta)$ where $\alpha$ (respectively $\beta$ ) denotes the application of internal (respectively external) negation. For instance, $(0,1)$ represents $Q(S, \neg P)$.

Since the corners of the modern square are defined by a starting protoform over which 3 transformations are applied, namely an internal negation, an external negation, and a composition of both, then any set of four protoforms in the 4-cube such that, applying these 3 transformations over one of them yields the 3 others defines a modern square too.

9 couples of negation operators $\left\{a_{1}, c_{1}, a c_{1}\right\} \times$ $\left\{a_{2}, c_{2}, a c_{2}\right\}$ can be chosen to define internal and external negation so as to find different modern squares. Since a modern square definition can start from any of the 16 vertices and dividing by the permutation of the corners due to the operation involutivity, then $(16 \times 9) / 4=36$ modern squares can be found in the 4-cube.
For instance, with the couple $\left(a_{1}, c_{2}\right)$, the modern square $Q P, \widehat{Q} P, Q \bar{P}, \widehat{Q} \bar{P}$ on the front face of the inner cube is determined. Another, less visible, square is given by the corners $Q P, \widehat{Q} P, Q \bar{P}, \widehat{Q} \bar{P}$. It must be remarked that the order of application of the negation operators differs between the modern square and the 4-cube. They nonetheless describe the same opposition relations, in a different order.

\section{ESTABLISHING ANTONYMY FROM DUALITY}

This section discusses the specific case of the $a_{1} c_{2}$ combination, that relates $Q P$ with $\widehat{Q} \bar{P}, \widehat{Q} P$ with $Q \bar{P}, \bar{Q} P$ with $\widehat{\bar{Q}} \bar{P}$ and so on.

\section{A. Definition}

The antonymy property (not to be confused with the antonym fuzzy negation, see Section V-C), desired in FLS [1], [9], [10], [26], states that:

$$
t(Q P)=t(\widehat{Q} \bar{P})
$$

e.g. "Most young people are tall" has the same truth value as "Few young people are not tall".

As discussed in Section VI-A, some pairs of negation protoforms can be equal depending on the properties of $Q$ and $P$, e.g. $Q P=\widehat{Q} P$ if $Q=\widehat{Q}$. Obviously, equal protoforms have equal truth values, so if $Q P=\widehat{Q} P$, then $t(Q P)=t(\widehat{Q} P)$.

The antonymy property, in turn, involves protoforms different in their definitions: when it is satisfied, $t(Q P)=$ $t(\widehat{Q} \bar{P})$, even if $Q P \neq \widehat{Q} \bar{P}$.

This relation deserves a specific attention for two reasons. First, it holds a particular role in the 4-cube of opposition since it is an equality on truth values, not on protoforms.

Second, it does not only involve $Q$ and $P$, but also the qualifier $R$ as it expresses a constraint on the truth value whose evaluation depends on $R$. It is satisfied when $R$ is always true, i.e. for protoforms " $Q x$ are $P$ ", but not in the general case " $Q R x$ are $P$ " [1], [6].

Below, we introduce the definition of the counting function negation, defining a final level of opposition in FLS, and a condition under which antonymy holds.

\section{B. Negation of the Counting Function}

The counting function $\nu(R, P)$ defined in Eq. (1) counts the number of elements in $R$ being $P$. Hence, $R$ can be seen as a restriction of the universe over which the number of $P$ are counted. So, denoting $|R|=\sum_{x} R(x)$, the interval of definition of $\nu$ is $[0,|R|]$ and does not depend on $P$. Hence, the negation of $\nu$ is denoted:

$$
\bar{\nu}(R, P)=n([0,|R|], \nu(R, P))=|R|-\nu(R, P)
$$

Semantically, the negation of the counting function depends on the number of considered elements, e.g. if 8 out of 10 satisfy $P$, the negation of this count is $10-8=2$. 


\section{Duality Property}

With the negation of the counting function, we introduce the duality property, satisfied for a counting function $\nu$ iff:

$$
\nu(R, \bar{P})=\bar{\nu}(R, P)
$$

The counting function is based on a t-norm (Eq. (1)). Among the four classical t-norms, drastic, Łukasiewicz, min and probabilistic, only the latter verifies duality. Indeed, denoting it $\top_{P}$ :

$$
\begin{aligned}
\nu(R, \bar{P}) & =\sum \top_{P}(R, 1-P)=\sum R(1-P) \\
& =\sum R-\sum \top_{P}(R, P) \\
& =|R|-\nu(R, P)=\bar{\nu}(R, P)
\end{aligned}
$$

It can be easily shown that this relation is not satisfied with the other three t-norms.

\section{Link between Duality and Antonymy Properties}

We now show that a counting function satisfying the duality property also satisfies the antonymy property.

If $Q$ is an absolute quantifier defined on $[0,|R|]$, then $\rho(R, Q)=1$ (Eq. 2) and :

$t(Q P)=Q(\nu(R, P))=Q(\bar{\nu}(R, \bar{P}))=\widehat{Q}(\nu(R, \bar{P}))=t(\widehat{Q} \bar{P})$

If $Q$ is a relative quantifier defined on $[0,1]$, then $\rho(R, Q)=1 /|R|$ and :

$$
\begin{aligned}
t(Q P) & =Q(\nu(R, P) /|R|)=Q(1-\nu(R, \bar{P}) /|R|) \\
& =\widehat{Q}(\nu(R, \bar{P}) /|R|)=t(\widehat{Q} \bar{P})
\end{aligned}
$$

Hence, the antonymy property can be satisfied with $\top_{P}$ on general protoforms " $Q R x$ are $P$ " and in this case, the duality relation in the 4-cube is an equality.

\section{CONCLUSION}

We proposed in this paper a thorough analysis of the different models of opposition between sentences of increasing complexity, from simple ones based on a single negation operator to quantified ones using generalised quantifiers and the several fuzzy negation operators defined in fuzzy logic.

As a result, we propose a complete view of all degrees of freedom inducing opposition as well as a graphical representation of all 16 negation forms, as a 4-cube of opposition. Additionally, introducing the notion of negation of the counting function, we establish a way to generally satisfy the antonymy property. Furthermore, we detail the relations between quantified sentences used in FLS and both the classical and modern squares of opposition.

Ongoing and future works cover a general definition of consistency in FLS based on the 4-cube, the definition and implementation of efficient algorithms ensuring non contradiction in FLS, and a further study of the counting function.

\section{REFERENCES}

[1] R. Yager, "A new approach to the summarization of data," Inf. Sci. (Ny)., vol. 28, no. 1, pp. 69-86, 1982.

[2] J. Kacprzyk and S. Zadrozny, "Computing With Words is an Implementable Paradigm: Fuzzy Queries, Linguistic Data Summaries, and Natural-Language Generation," IEEE Trans. Fuzzy Syst., vol. 18, no. 3, pp. 461-472, 2010.

[3] J. Kacprzyk and S. Zadrozny, "Comprehensiveness and interpretability of linguistic data summaries: A natural language focused perspective," in Proc. IEEE SSCI CIHLI'13, pp. 33-40, 2013.

[4] M.-J. Lesot, G. Moyse, and B. Bouchon-Meunier, "Interpretability of Fuzzy Linguistic Summaries," Fuzzy Sets Syst., 2014.

[5] J. Kacprzyk and S. Zadrozny, "Protoforms of Linguistic Data Summaries: Towards More General Natural-Language-Based Data Mining Tools," in Soft Comput. Syst., pp. 417-425, 2002.

[6] L. Zadeh, "A computational approach to fuzzy quantifiers in natural languages," Comput. Math. with Appl., vol. 9, pp. 149-184, 1983.

[7] B. Bouchon-Meunier and G. Moyse, "Fuzzy Linguistic Summaries: Where Are We, Where Can We Go?," in Proc. of CIFEr'12, pp. 317 324,2012

[8] L. Zadeh, "The concept of a linguistic variable and its application to approximate reasoning - I," Inf. Sci. (Ny)., vol. 8, pp. 199-249, 1975.

[9] I. Glöckner, "DFS - An Axiomatic Approach to Fuzzy Quantification," tech. rep., Universität Bielefeld, 1997.

[10] I. Blanco, M. Delgado, M. Martín-Bautista, D. Sánchez, and M. Vila, "Quantifier guided aggregation of fuzzy criteria with associated importances," in Aggreg. Oper., pp. 272-287, 2002.

[11] M. Delgado, M. Ruiz, and D. Sánchez, "A restriction level approach to the representation of imprecise properties," Proc. of IFSAEUSFLAT'09, pp. 153-159, 2009.

[12] T. Martin, "The X-mu representation of fuzzy sets - Regaining the excluded middle," in Proc. of UKCI'13, pp. 67-73, 2013.

[13] M. Delgado, M. Ruiz, D. Sánchez, and M. Vila, "Fuzzy quantification: a state of the art," Fuzzy Sets Syst., vol. 242, pp. 1-30, 2014.

[14] L. Horn, A Natural History of Negation. Center for the Study of Language and Information, 2002.

[15] D. Westerstahl, "Classical vs. modern Squares of Opposition, and beyond," in Sq. Oppos. A Gen. Framew. Cogn., pp. 195-229, 2012.

[16] R. Blanché, Les structures intellectuelles, essai sur l'organisation systématique des concepts. J.Vrin, 1966.

[17] D. Dubois and H. Prade, "From Blanché's Hexagonal Organization of Concepts to Formal Concept Analysis and Possibility Theory," Log. Universalis, vol. 6, no. 1-2, pp. 149-169, 2012.

[18] A. Moretti, "From the "logical square" to the "logical poly-simplexes". A quick survey of what happened in between.," in New Perspect. Sq. Oppos., pp. 1-21, 2011.

[19] A. Mostowski, "On a Generalization of Quantifiers," Fundam. Math., vol. 44, no. 1, pp. 12-36, 1957.

[20] J. Barwise and R. Cooper, "Generalized quantifiers and natural language," Linguist. Philos., vol. 4, no. 2, pp. 159-219, 1981.

[21] M. Brown, "Generalized quantifiers and the square of opposition," Notre Dame J. Form. Log., vol. 25, no. 4, pp. 303-322, 1984.

[22] P. Peterson, "On the logic of "Few", "Many" and "Most"," Notre Dame J. Form. Log., vol. 20, no. 1, pp. 155-179, 1979.

[23] V. Novák, "A formal theory of intermediate quantifiers," Fuzzy Sets Syst., vol. 159, no. 10, pp. 1229-1246, 2008.

[24] P. Murinová and V. Novák, "Analysis of generalized square of opposition with intermediate quantifiers," Fuzzy Sets Syst., vol. 242, pp. 89-113, 2014.

[25] A. R. de Soto and E. Trillas, "On Antonym and Negate in Fuzzy Logic," Int. J. Intell. Syst., vol. 14, no. 3, pp. 295-303, 1999.

[26] S. Barro, A. Bugarín, P. Cariñena, and F. Díaz-Hermida, "A framework for fuzzy quantification models analysis," IEEE Trans. Fuzzy Syst., vol. 11, no. 1, pp. 89-99, 2003. 